discover if using a cut off value of $>100$ was adequate in diagnosing IBD.

Method A retrospective analysis was conducted for all patients in Chesterfield who had a FC requested by their GP between July and December 2017. The notes of those referred and seen in clinic were then analysed.

Results 498 patients had a FC performed by their GP. 107 patients were seen in clinic, 58 were female and 49 were male. Ages ranged from 16 years to 89 years with FC results from $<8$ to 1086 . 9 patients in total were diagnosed with IBD. If a cut off FC $>100$ were used; 42 of these patients would have been referred with 6 being diagnosed with IBD. 65 would not have been referred but 3 would have IBD, giving a sensitivity of $66.67 \%$ (95\% CI $29.93 \%$ to $92.51 \%)$ and a specificity of $63.27 \%$ (95\% CI $52.93 \%$ to $72.78 \%$ ). The positive predictive value of our test would therefore be 14.29\% (95\% CI $8.93 \%$ to $22.07 \%$ ) with negative predictive value of $95.38 \%$ (95\% CI $89.02 \%$ to $98.14 \%$ ).

Conclusion Our study has shown that using a cut off value of $>100$ for FC values will result in a lower sensitivity when compared to data from other groups. However, a larger cohort of patients will need to be retrospectively analysed to determine whether a cut off value for FC of 100 should be used or if it should be lowered in order to improve sensitivity and specificity in diagnosing patients with IBD. In addition, we have discovered that FC testing is being incorrectly performed in patients who meet other diagnostic pathways and so further advice and guidance needs to be given regarding its use.

\section{PTU-091 RIGHT HEMICOLECTOMY IN CROHN'S DISEASE - ARE WE FOLLOWING ECCO GUIDELINES?}

Sarah Flatley*, lan Reilly. Countess of Chester Hospital, Chester, UK

\subsection{6/gutjnl-2019-BSGAbstracts.450}

Introduction Despite recent advances in medical therapy, approximately $80 \%$ of patients with Crohn's disease will require surgery within 20 years of diagnosis. Surgery is not curative and clinical and/or endoscopic recurrence occurs in the majority of patients. Correct pre-operative planning and post-operative care has a major impact on the outcome of such treatment. The aim of this study was to compare local practice in the Countess of Chester Hospital with published ECCO guidelines, to identify opportunities to improve care.

Methods This was a retrospective analysis of all patients with Crohn's disease that underwent a right hemicolectomy between January 2013 and December 2017 at the Countess of Chester Hospital. Patients were identified by obtaining a list of right-hemicolectomy specimens from the histopathology department. Patient notes were reviewed to ascertain patient demographics, pre- and post-operative treatment, and followup endoscopy.

Results A total of 14 patients were identified for this study. Age range was $2-5$ with a male to female ratio of $4: 3$. The Montreal classification varied widely. Only $8 / 14$ (57\%) of patients had received an immunomodulator pre-operatively and 2/14 (14\%) were on a biologic. 10/14 (71\%) of patients were not given either an immunomodulator or biologic postoperatively. Follow-up endoscopy occurred in 7/14 (50\%) patients. 3 of these were within 6 months and 4 were within 12 months of the operation date. $3 / 7$ (42.9\%) of patients had evidence of recurrence on endoscopy. None of the patients had a Rutgeerts' score generated.

Conclusions This study demonstrates the variability of management of patients with Crohn's disease post-operatively. Our results show that over $60 \%$ of patients were not on any medical treatment pre-operatively. In addition, over $75 \%$ of patients continued to remain on no maintenance after their operation. Only 50\% of patients underwent a follow-up endoscopy within 12 months of their operation date and a Rugeerts' score was not generated in any of these patients.

\section{PTU-092 EXPLORING ACCESS TO SECONDARY CARE SERVICES FOR PATIENTS WITH ESTABLISHED INFLAMMATORY BOWEL DISEASE}

Sharon Gethins*, Sanjeev Pattni, Richard Robinson. University Hospitals Of Leicester, Leciester, UK

\subsection{6/gutjnl-2019-BSGAbstracts.451}

Introduction The Inflammatory Bowel Disease (IBD) Standards of Care recommend that defined arrangements are in place to allow for direct admission or assessment to a gastroenterology unit for patients with UC or Crohn's. Currently in Leicester there is no facility for rapid access or direct admission to inpatient GI services. The aim of this study was to establish how patients with (IBD) accessed secondary-care services when admission was required.

Methods Consecutive patients admitted to gastroenterology with an established diagnosis of IBD were asked to complete a questionnaire exploring their point of access, admission process, waiting times and treatment during admission before their discharge from hospital

Results 50 patients were recruited (30 UC,19 CD,1 indeterminate) First point of access after admission was recommended included 30 pts arriving at $\mathrm{A} \& \mathrm{E}, 11$ via medical admission unit, 7 directly via gastroenterology services and 2 to other departments.

Other pathways once admitted involved 14 pts transferring to 2 -wards and 29 pts to 3 wards.

32 patients were commenced Intravenous steroids in first 24 hours, 8 patients waited longer than 24 hours. 8 patients did not commence any treatment relating to an acute exacerbation of IBD

Conclusions Patients were admitted to a number of different wards via a variety of routes. Many were waiting for long periods of time \& some patients did not require hospital admission. As a result of this evaluation we have established a 'Hot Clinic'

\section{PTU-093 EVALUATING A NURSE LED INFLAMMATORY BOWEL DISEASE HOT CLINIC}

Sharon Gethins*, Sanjeev Pattni, Richard Robinson, Tracey Duckett, Jodie Walton, Kelly Hiatt. University Hospitals Of Leicester, Leciester, UK

\subsection{6/gutjnl-2019-BSGAbstracts.452}

Introduction Inflammatory Bowel Disease (IBD) can be an unpredictable condition with relapses requiring escalation of therapy and possible admission to hospital. The IBD Standards of Care recommend that patients with Crohn's disease and Ulcerative colitis have rapid access to specialist doctors and 